\title{
TELEREHABILITATION IN SOUTH AFRICA - IS THERE A WAY FORWARD?
}

\author{
MAURICE MARS, MBCHB, MD \\ Department Of Telehealth, Nelson R. Mandela School Of Medicine, \\ UNIVERSITY OF KWAZULU-NATAL, DURBAN, SOUTH AFRICA
}

\section{ABSTRACT}

South Africa, like the rest of sub-Saharan Africa, has a disproportionate burden of disease and a shortage of health professionals. Telemedicine has been identified as a possible way of overcoming part of the problem but telemedicine has not been widely adopted. In the public sector hospitals in South Africa which serve $82 \%$ of the population there are 2.5 physiotherapists and 2 occupational therapists per 100,000 people served. The extent of telerehabilitation in South Africa is unknown. A literature review of telerehabilitation found no papers from South Africa. A survey of the heads of university departments of physiotherapy, occupational therapy and speech and language pathology revealed limited knowledge of telerehabilitation. Telerehabilitation services are confined to follow-up of patients at some institutions by telephone, fax or email. There is need to raise awareness among therapists if telerehabilitation is to become a reality in South Africa. Future actions are outlined.

Keywords: South Africa, sub-Saharan Africa, telerehabilitation, telemedicine

\section{INTRODUCTION}

Health care delivery faces a common problem across the world - money or lack thereof. In the developed world healthcare is either too expensive or becoming too expensive as populations age, require more services and chronic disease increases. In the developing world where national budgets are small and the percentage of direct government expenditure on health is also small, there is insufficient money to provide adequate services (World Health Organization, 2010). Is telerehabilitation a viable or relevant option in the developing world? The question will be reviewed from the viewpoint of South Africa, which is in many respects a microcosm of sub-Saharan Africa and the developing world.

\section{SOUTH AFRICA}

South Africa is a relatively large country of $1,221,037$ $\mathrm{km} 2$, lying at the southern tip of the continent of Africa. It has an estimated total population of 49.9 million people who are almost equally divided between rural (46\%) and urban areas (Health Systems Trust, 2011). The country has been a constitutional democracy since 1994 and is divided into nine Provinces, each with a Provincial Legislature and their own Department of Health (DOH). There are 11 official languages. With a gross domestic product of
US\$354 Billion it is one of the largest and most developed economies in Africa and accounts for about a quarter of the entire GDP of the continent (World Bank, 2011).

South Africa has recently been invited to join the emerging nations group of Brazil, Russia, India and China and is serving a second term on the United Nations Security Council. Despite this newfound status, poverty is still rife and the official unemployment rate is $24 \%$ with an unofficial unemployment rate that is in the range of 3545\% (Statistics South Africa, 2010). Forty-seven percent of its people live below the poverty datum line, and $26 \%$ below US\$1 (purchasing power parity) per day (Armstrong, Lekezwa, \& Siebrits, 2008; World Health Organization, 2010). There is significant maldistribution of wealth. South Africa has one of the highest GINI coefficients in the World of $57.8 \%$. This is the measure of the distribution of income among individuals or households in the country. It is ranked 110th in the world on the Human Development Index (United Nations Development Program, 2010). The country has both wealth and poverty and the poor form a microcosm of the rest of sub-Saharan Africa.

Issues affecting eHealth and the current status of telemedicine in South Africa need to be described to better understand the context within which telerehabilitation may occur. 


\section{FACTORS AFFECTING HEALTHCARE DELIVERY}

In sub-Saharan Africa the burden of disease is great, there is extreme poverty, a shortage of health workers and a rapidly growing population (United Nations Department of Economic Social Affairs Population Division, 2009). The World Health Report of 2006 stated that, "...Africa has $24 \%$ of the burden (of disease), but only $3 \%$ of the health workers commanding less than $1 \%$ of world health expenditure." and "The exodus of skilled professionals in the midst of so much unmet health needs places Africa at the epicentre of the global health workforce crisis." (World Health Organization, 2006b). These issues need to be examined in the South African context.

\section{BURDEN OF DISEASE}

The burden of disease is great. The prevalence of HIV in adults aged 15 years or older in South Africa is $18.1 \%$ and until recently South Africa had more HIV positive people than any other nation (World Health Organization, 2010). Child mortality under the age of 5 years is $47 / 1,000$ births and average life expectancy is 52 years (Health Systems Trust, 2011). The incidence of tuberculosis is $960 / 100,000$ and rising as is the incidence of multi-drug resistant (MDR) and extensively drug resistant (XDR) tuberculosis (World Health Organization, 2010).

\section{HeAlth Care Systems And FUnding}

Healthcare is provided by the State in Public Hospitals where fees are based on income and all health professionals are salaried, and by the private sector with patients either self funded or partially covered by medical insurance. Public health services and budgets in South Africa are devolved from the National Treasury to the National $\mathrm{DOH}$ and the nine Provincial DOHs. Some primary healthcare services are provided by municipalities. The annual healthcare spend by Government is US\$206 per capita per annum. This far exceeds the average Government expenditure on health in sub-Saharan Africa of US $\$ 114$ per capita, per annum with a median expenditure of US $\$ 34$ per capita, per annum (World Health Organization, 2010). There is a major inequality in healthcare funding, with $60 \%$ of the total health-spend in South Africa consumed by $18 \%$ of the population who have medical insurance and who access the private medical sector (South Africa Info, 2011).

\section{HUMAN RESOURCES}

By sub-Saharan Africa's standards, where 31 countries have 10 doctors or fewer per 100,000 people, South Africa with 77 doctors per 100,000 people is well supplied but this should be compared with Germany, the USA, and the United Kingdom, where there are 300, 260, 230 doctors per 100,000 people respectively (World Health Organization, 2010). There is a shortage of 4 million health professionals in Africa (World Health Organization, $2006 \mathrm{~b})$. The distribution of doctors in the South Africa is skewed with the Public Hospital sector served by 27.3 doctors per 100,000 people and 10.0 specialists per 100,000 people and the private sector by 57 specialists per 100,000 . On average, $49 \%$ of the medical posts in the public health sector are vacant (Health Systems Trust, 2011).

Qualified therapists register to practice through the relevant Professional Board of the Health Professions Council of South Africa and are entitled to practice anywhere in the country. Data on the number of therapists in Africa are limited. The World Federation of Occupational Therapists lists the total number of practising occupational therapists in seven African countries. This ranges from 17 in Mauritius to 3,166 in South Africa and from 0.2 per 100,000 in Tanzania to 6.6 per 100,000 people in South Africa (World Federation of Occupational Therapists, 2011). The World Confederation of Physical Therapy provides data for 15 member nation African countries with the number of physiotherapists ranging from 15 in Swaziland to 3,254 in South Africa and from 0.1 per 100,000 people in Ethiopia and Ghana to 6.7 per 100,000 people in South Africa (World Confederation of Physical Therapists, 2011). The problem with these figures is that they reflect only the membership of the National Physical Therapy Associations and not the total number of physiotherapists registered to practice. In South Africa, there were 5,777 physiotherapists registered with the Health Professions Council in 2010, which is 11.5 physiotherapists per 100,000 people suggesting that $40 \%$ of physiotherapists are not members of the South African Society for Physiotherapy (Health Systems Trust, 2011). Whether this is true for the other countries is not known.

These numbers are low by developed world standards but as with the provision of doctors mask further disparities. The Public Sector health services are served by only 1,009 physiotherapists and 838 occupational therapists, 2.5 and 2.0 per 100,000 people respectively (Health Systems Trust, 2011). The 9 million people who have access to private or insurance funded care are relatively well provided for with 53 physiotherapists and 30 occupational therapists per 100,000 people. This is comparable to the density of 52 and 39 physical therapists and occupation therapists per 100,000 people in Canada (Ontario Hospital Association, 2011). As the population of sub-Saharan Africa is forecast to double by 2050 (United Nations Department of Economic Social Affairs Population Division, 2009) and the production of health professionals is already failing to meet demand, the ratio of therapists to population will therefore continue to worsen. 


\section{TELEMEDICINE IN SOUTH AFRICA}

eHealth and telemedicine have been proposed as a possible way of addressing some of the health problems of the developing world. Resolution WHA58.28 of the World Health Assembly of 2005 called on member nations to develop long term eHealth strategic plans, provide necessary telecommunications infrastructure for eHealth and establish national centres of excellence (Committee A, 2005). The WHO Global Observatory for eHealth's report of 2006 suggested that, "It may be time to put forth the concept of 'eHealth for all by 2015' as an addendum to the Millennium Development Goals." (World Health Organization, 2006a).

The South African Government had an enlightened view of telemedicine and launched Phase One of the National Telemedicine Project in 1998. This involved 28 sites in Public Hospitals in teleradiology, antenatal teleultrasonography, telepathology and tele-ophthalmology (Gulube \& Wynchank, 2002). The project was developed within the National $\mathrm{DOH}$ and was devolved to the various Provincial DOHs on roll-out. It soon failed because of lack of buy-in, limited or absent budgeting by the Provincial DOHs, failure to appoint people to manage telemedicine, limited bandwidth and poor change management. The second and third phases of the project were never funded or implemented. It has taken 10 years for the Government to express interest again in telemedicine which is now seen as a national priority in Health. A National eHealth Policy and a new National Telemedicine Strategy are being developed. The initial focus of Government will be on teleradiology.

Despite the failure of the national project, telemedicine projects have been implemented in several Provinces through Provincial DOHs and University Medical Schools and Health Faculties. There are however few sustained programmes. In KwaZulu-Natal, small services in teleophthalmology and teledermatology have been running for 8 years (Mars, 2007) and recently services have been started in tele-orthopaedics and telepsychiatry. In several Provinces CT scanners have been linked to Academic Teaching Hospitals for teleradiology (Corr, 1998) and teleradiology for neurosurgery has been effectively incorporated into neurosurgical practice in two provinces (Jithoo, Govender, Corr, \& Nathoo, 2003). The Medical Research Council of South Africa has a Telemedicine Lead Programme which has been involved in the development of a telemedicine work station designed for use in primary care facilities (Molefi, 2006).

In the private sector teleradiology is the norm, telepathology is growing and there are anecdotal reports of the use of tele-ecg and medical apps. Wilderness telemedicine using blends of mobile phone, radio, Skype and email has evolved to provide emergency telemedicine cover to isolated game lodges and parks.

Interest in telemedicine has grown to the point that the South African Telemedicine Association was formed in
2010. Membership includes clinicians in the private and public sectors, industry, academia, government agencies, non-governmental organizations and technical and clinical support staff.

\section{OBSTACles TO EHEALTH}

There are several barriers to the implementation of telemedicine solutions in the Public Sector and telemedicine in general in South Africa which pertain to all of sub-Saharan Africa. Telemedicine requires information and communication technologies, their associated infrastructure, and power. Power supply is irregular in much of Africa and is an already growing problem in South Africa.

Fixed telephone line penetration in South Africa is 8.6\%, Internet penetration is $10.8 \%$ and connectivity is limited. Fixed broadband penetration is less than 1\% (International Telecommunications Union, 2011; Minwatts Marketing Group, 2011). The very low broadband penetration severely limits synchronous IP based videoconferenced telemedicine. In KwaZulu-Natal the majority of the Public hospitals are allocated only $128 \mathrm{kbs}-1$ total bandwidth for all hospital activities. It is not that the bandwidth is not available, but that the Provincial DOH cannot justify the cost of additional bandwidth. Paradoxically, this necessitates the use of more costly ISDN lines for videoconferencing.

The cost of telecommunication services is very high. The International Telecommunications Union has developed an ICT Price Basket and ranks 161 countries on the basis of a set of standardized fixed phone line telephony, mobile cellular and broadband services and describes these in terms of relative cost, expressed as a percentage of the average monthly Gross National Income (GNI) per capita. Seventeen of the twenty most expensive countries are in sub-Saharan Africa. The cost of a monthly broadband subscription and $1 \mathrm{~Gb}$ of data exceeds the monthly GNI per capita in 19 African countries and averages $500 \%$ of GNI per capita. In South Africa the cost is $5.5 \%$ of monthly GNI per capita and the cost of the same services in the United Kingdom and the USA is $0.6 \%$ and $0.5 \%$ of $\mathrm{GNI}$ respectively (International Telecommunications Union, 2011). Likewise telephone costs are higher. With such low Internet penetration and the high communication costs, email based store and forward telemedicine is also expensive and patient centric solutions like web portals and web 2.0 are currently irrelevant for most of the continent.

Mobile telephone penetration is growing rapidly and is now in the region of $41 \%$ in sub-Saharan Africa and $95 \%$ in South Africa (International Telecommunications Union, 2011). mHealth is the new buzz word, with mHealth seen as the new way of facilitating provision of telemedicine services. The widespread use of smart phones and apps is some way off for Africa. There are however a number 
of examples of mobile phone use in Africa for data collection and improving antiretroviral and TB medication compliance through text messaging and automated phone calls (Cell-Life, 2011; Simpill, 2011). Call costs are relatively high and most people buy airtime in small denominations. Currently there is reluctance among the poor to use mobile phones for non-emergency medical services.

\section{TELEREHABILITATION}

Telerehabilitation is particularly appealing in subSaharan Africa where trauma, both accidental and conflict related, contributes significantly to the burden of disease and pathology is often advanced before medical attention is sought. With $63 \%$ of the population of sub-Saharan Africa living in rural areas, it also offers the chance of access to professional expertise that is usually situated in major cities (United Nations Department of Economic and Social Affairs, 2010).

The aims of this study were to conduct a literature review to determine the extent and nature of telerehabilitation reported in South Africa and subSaharan Africa and to survey the heads of University departments of Physiotherapy, Occupational Therapy and Speech Therapy in South Africa to determine telerehabilitation activities in their departments and in South Africa.

\section{METHODS}

Searches of the electronic databases Medline, CINAHL and African Index Medicus were undertaken. For Medline and African Index Medicus the search terms were:

((“Physical Therapy Modalities”[Mesh] OR "Physical Therapy (Specialty)"[Mesh]) OR "Physical Therapy Department, Hospital"[Mesh]) OR ("Occupational Therapy"[Mesh] OR "Occupational Therapy Department, Hospital"[Mesh]) OR "Speech Therapy"[Mesh] AND ("Telemedicine"[Mesh] OR "Telecommunications"[Mesh] OR "Remote Consultation" [Mesh] OR "Computer Communication Networks"[Mesh] OR "Electronic Mail”[Mesh] OR "Videoconferencing"[Mesh] OR

"Telerehabilitation"[All Fields]).

CINAHL searches were made using the following search terms:

(MH "Speech Therapy+") AND ((MH "Telemedicine+") OR "ehealth" OR (MH "Telehealth+") OR (MH

"Videoconferencing") OR "Internet" OR "telerehabilitation"

OR (MH "Electronic Mail"))

("Physiotherapy" OR (MH "Physical Therapy+") OR (MH

"Home Physical Therapy")) AND ((MH "Telemedicine+")

OR "ehealth" OR (MH "Telehealth+") OR (MH
"Videoconferencing") OR "Internet" OR "telerehabilitation" $\mathrm{OR}(\mathrm{MH}$ "Electronic Mail"))

(MH "Occupational Therapy+") AND ((MH

"Telemedicine+") OR "ehealth" OR (MH "Telehealth+")

OR (MH "Videoconferencing") OR "Internet" OR

"telerehabilitation" OR (MH "Electronic Mail"))

Additional searches for each search in Medline with AND ("Africa" [Mesh]) and in CINAHL with AND (MH "Africa+") OR "Africa" OR (MH "Africa South of the Sahara+") OR (MH "Africa, Central+") OR (MH "Africa, Eastern+") OR (MH "Africa, Northern+") OR (MH "Africa, Southern+") OR (MH "Africa, Western+") OR (MH "South Africa") were undertaken.

The abstracts of all papers found were reviewed by two independent reviewers, and all papers with an English abstract reporting clinical data, validation studies, reviews and economic assessments of some form of telerehabilitation were identified. Papers reporting proposed studies, the development of technologies including garment sensors, laboratory assessments and robotic use in hospital rehabilitation settings were excluded. Where there were disputes about paper suitability these were resolved through discussion and if agreement was not reached the paper was included.

A questionnaire was sent by email to the 21 heads of University Departments of Physiotherapy, Occupational Therapy and Speech Therapy of the eight Medical Schools in South Africa. It contained 15 questions about their knowledge of telerehabilitation activities in their discipline in South Africa. The questions related to use of telephone, fax, Internet, email, Skype or other VolP solution, videoconferencing, computer programmes, home based devices, robotics and virtual reality. Where responses were not obtained within one week the recipients were telephoned and the questionnaire completed if possible. The decision to survey academic heads of departments was based on the assumption that as academic leaders, educators and researchers in their fields in South Africa they would have knowledge of current clinical use of and research endeavors in telerehabilitation. 


\section{RESULTS}

The initial Medline search returned 481 papers. The additional searches on Africa and individual countries returned 1 paper. No papers were found in the African Index Medicus. The CINAHL database searches returned 27 papers for Speech Therapy, 345 for Physical OR Physiotherapy, and 83 for Occupational Therapy. One paper was found on Africa but was not relevant. After reviewing the papers 101 papers met the entry criteria and of these none related to Africa.

The telemedicine / telerehabilitation component of the search strategy was run independently in Medline and returned 89,996 papers. A search on telerehabilitation returned 146 papers $(0.16 \%)$ of all papers on telemedicine / telerehabilitation and the 481 papers relating to telemedicine/telerehabilitation in Physical, Occupational and Speech Therapy constitute $0.5 \%$ of the telemedicine/ telerehabilitation search results.

Response to the questionnaire was very limited. Of the eight medical schools two did not respond at all. Nine of the remaining sixteen heads of department responded to the email and the others were contacted telephonically. The telephone is used for patient follow up in four departments one of which also uses the phone to provide support to community service therapists. One provides support to patients by telephone, fax and email. No use is made of store and forward telerehabilitation. Skype is used to support research in one department and to communicate with community service therapists by another. One department uses videoconferencing for staff support and education. Reminders for group therapy sessions are sent by SMS by one department and none uses Internet based instant messaging or chat, specialised computer software, computer games, specialised devices, robotics or virtual reality for rehabilitation at a distance.

\section{DISCUSSION}

Telerehabilitation constitutes a very small part of the literature on telemedicine and there are no papers about telerehabilitation in Africa. While this may reflect the problem that African researchers often have difficulty in publishing in internationally indexed journals, the African Medicus Index also failed to produce any papers. If telerehabilitation is being practiced in sub-Saharan Africa it is yet to be reported in mainstream literature.

The survey reveals very little telerehabilitation in the University Departments. Patient related use of information and communication technology is limited to the telephone, fax or email for patient follow-up in four departments. No use is made of videoconferencing or web based applications for patient management. Although the response rate was poor, respondents were asked to report what they knew of the use of telerehabilitation in South Africa.

For telerehabilitation to be successfully implemented in South Africa there needs to be awareness of telerehabilitation and the scope of practice. The academic teaching departments are largely unaware of telerehabilitation. Several people gave unsolicited responses that they thought telerehabilitation was a good idea but that they didn't know anything about it. Those that have used videoconferencing, Skype, email and telephony have been driven by local need and availability of infrastructure. These early and albeit probably unknowing early adopters need to become the "champions" of telerehabilitation. The newly formed South African Telemedicine Association (SATMA) has identified telerehabilitation as an area for development. The limited use and awareness of telerehabilitation by the academic departments has led to a web based survey of the membership of the South African Physiotherapy Society and the Private Practitioners Association on current understanding and use of telerehabilitation and their potential needs. Similar surveys are being organised for occupational and speech therapy.

A telerehabilitation special interest group (SIG) is to be formed in SATMA, along the lines of the Telerehabilitation SIG of the American Telemedicine Association (ATA) once the electronic surveys have been completed and relevant enthusiasts have been identified. In the interim a working group of interested members of the academic departments is working towards raising awareness of telerehabilitation through presentations at national conferences and regional meetings, publication of review papers in local journals and presentations to University Departments. Longer term goals are envisaged to include development of position statements on the use telerehabilitation by the various national associations and societies, the development of guidelines for telerehabilitation, interaction with the respective Boards of the Health Professions Council on regulatory and ethical issues and the Association of Health Insurers on remuneration. As there is lack of experience in telerehabilitation the formulation of guidelines will need to draw from existing guidelines such as those being developed by the ATA Telerehabilitation SIG. The approach that we have taken in other disciplines is to modify guidelines from other countries to suit the local environment, infrastructure and regulations and then submit these to national associations for further input and subsequent adoption. We believe that it is important for a discipline to take ownership of the "tele" aspect of its practice rather than have government regulators dictate what is best for the discipline (Kekana, Noe, \& Mkhize, 2010; Mars \& Jack, 2010). This is done to overcome the one size fits all approach of regulators.

The surveys will, it is hoped, identify perceived needs. Past experience in implementing telemedicine in other disciplines suggests that there will be some commonality, but there will be different needs and solutions in the 
private, public and academic sectors. While clinical need is great, especially in the rural areas, we have found that it is often difficult to start sustainable telemedicine services as health practitioners are reluctant to participate in telemedicine programmes that add extra steps to the normal workflow. In a salaried environment which is already understaffed there is little incentive to take on extra work. This is particularly so if there is no on-site telemedicine coordinator and limited or no technical support. In our region, we are yet to overcome this shortcoming as it requires either new posts or changes to existing job descriptions. While the Provincial Government acknowledges the problem it does not see this as priority when faced with high infant mortality, HIV prevalence and an increasing incidence of TB.

We have found that despite reluctance to participate in clinical telemedicine services, health professionals in rural areas readily participate in videoconferenced postgraduate education and continuing professional development (CPD) session (Mars, 2009). In KwaZuluNatal videoconferencing takes place using ISDN lines to connect at $128 \mathrm{kbs}-1$. In 2010 over 1400 hours of interactive videoconferenced teaching in a variety of disciplines took place from the Nelson R. Mandela School of Medicine (NRSM) to peripheral sites. This generated over 75,000 CPD points. In the absence of a videoconferencing bridge, simultaneous participation is limited to five sites. Thirty public hospitals in the province have videoconference units but not all are utilised for education. This is approximately half of all the videoconference units in public hospitals in the country.

In one rural area the physiotherapists at two hospitals established a clinical discussion group by videoconferencing. Apart from overcoming isolation, this also provided mentoring and support for newly qualified therapists who have to serve a year of community service before being allowed to practice independently. The South African Society for Physiotherapy has identified senior practitioners who provide telephonic support for community service physiotherapists. With Skype and desktop videoconferencing this can be adapted to a mentoring service. We have used videoconferencing to provide lectures and tutorials to undergraduate and postgraduate students undertaking clinical training at regional and district hospitals and to extend access to postgraduate coursework qualifications to students in rural areas. There are several coursework masters programmes designed to be offered by videoconferencing and a learning management system. This concept can be developed by academic departments for relevant postgraduate programmes and should not be limited to South Africa. The NRSM already offers postgraduate programmes in both telemedicine and medical informatics to students in other African countries through videoconferencing and Skype to assist in overcoming the shortage of educators in these disciplines in most African countries (Mars, 2009). It should be remembered that when there is a shortage of health professionals there is also a shortage of health professionals to teach health professionals.

In the public sector, those few hospitals with videoconferencing facilities will be able to offer some face to face clinical and mentoring services. For those without videoconferencing units Skype and desktop videoconferencing are going to be dependent on available bandwidth, local policy on Web access, and local firewall policy on communication outside the Government or Provincial Health virtual private networks (VPN). The State Information Technology Agency has set up a National Telemedicine VPN to enable telemedicine practice between provinces. It is currently limited to public hospital employees. If the relatively well staffed private sector is to assist the public sector in overcoming its shortage of therapists through telerehabilitation services the access policy will need revision.

The private sector treats the more affluent. Internet access is common in this group and mobile phones ubiquitous. Existing Web based assessment tools using, audio, video, images and text will be of use. For physiotherapists, the ability to perform assessments of range of movement and muscle power over the Web, supplemented by Skype or telephonic contact will be of interest. Store and forward telerehabilitation using pictures taken and sent from mobile phones offers potential for more regular assessment of patients performing self supervised home exercises.

The use of store and forward mHealth based telerehabilitation is appealing but needs an understanding of mobile phone use, the sophistication of the phones and signal coverage. If image based it is reliant on the phone having a camera. A recent sample of 247 patients in our region has shown that all patients attending urban private doctors have a mobile phone compared to $87 \%$ of patients attending rural public hospitals. Of these, $91 \%$ of the phones of the urban private patients and $53 \%$ of the rural patients' phones have a camera. Airtime is usually obtained on a pay as you go basis for $97 \%$ of rural patients and $73 \%$ of private patients. As a result telephones are not always functional and $35 \%$ of urban private patients and $50 \%$ of rural patients have been without airtime for more than a week in the past year. Power is also an issue with $15 \%$ of people unable to keep their phone regularly charged.

Despite these shortcomings, with mobile phone signal availability covering approximately $95 \%$ of the country, mobile phone use and the development of protocols and standards for mHealth facilitated telerehabilitation should be an area of research in South Africa. Smart phones and their health applications will be limited by cost until smart phones become the industry standard. What is needed are simple text and image based solutions which will be applicable to the rest of sub-Saharan Africa and the developing world. 
While there has been interest in the concept of telemedicine there has until recently been little political will to make it a reality. Within Africa neither the New Partnership for Africa's Development (NEPAD) Health Strategy nor the Health Strategy of the African Union (AU) for 2007 to 2015 mentions eHealth, telemedicine or telehealth (African Union, 2007; Nepad, 2009). The African Health Ministers Conference of 2009 did however call on the AU to work towards Harmonization of eHealth and Telemedicine in Africa but little has been done. The recent adoption of a resolution by the African Region of the WHO urging countries to move towards eHealth may catalyse change (World Health Organization African Region, 2011). In South Africa the government has made telemedicine a priority and a Ministerial Advisory Committee on Medical Technology which incorporates telemedicine has recently been formed. Currently lacking in South Africa are guidelines for the practice of telemedicine. Draft regulations for telemedicine to be appended to the Health Act were proposed in 2008. These were based on the Malaysian Telemedicine Act of 1997 and were very restrictive. They were never enacted. In 2008 the Health Professions Council developed ethical guidelines for the practice of telemedicine in South Africa (Kekana, et al., 2010). These were based on a poor definition of telemedicine and proposed that written consent be mandatory for all aspects of telemedicine including the use of the telephone. To date these have not been published. Telemedicine and telerehabilitation needs to be supported by enabling legislation and regulations that take into account the current constraints of inadequate infrastructure, the shortage of personnel and the need to overcome this shortage through international crossborder practice (Mars \& Jack, 2010; Mars \& Scott, 2010).

The obstacles to eHealth implementation and use in South Africa are many and there are few sustained telemedicine programmes. The current environment may not yet be conducive to the implementation and uptake of telerehabilitation but with awareness campaigns, the formation of a SIG and the development of a telerehabilitation research agenda this should change.

eHealth in Africa is approximately 10 years behind the developed world. The issue is whether it is possible to catch up, or will there be an ever growing eHealth digital divide? Poverty and the burden of disease will be major determinants both in South Africa and sub-SaharanAfrica.

\section{REFERENCES}

1. African Union. (2007). Africa health strategy: 2007-2015.

2. Armstrong, P. L., Lekezwa, B., \& Siebrits, F. K. (2008). Poverty in South Africa: A profile based on recenthousehold surveys. EconPapers, (4), 26. Retrieved from http:// EconPapers.repec.org/RePEc:sza:wpaper:wpapers52

3. Cell-Life. (2011). Cell Life. from http://www.cell-life.org/

4. Committee A. (2005). World Health Assembly eHealth Resolution (WHA 58.28).

5. Corr, P. (1998). Teleradiology in KwaZulu-Natal. A pilot project. S.Afr.Med.J., 88(1), 48-49.

6. Gulube, S. M., \& Wynchank, S. (2002). The national telemedicine system in South Africa--an overview and progress report. S.Afr.Med.J., 92(7), 513-515.

7. Health Systems Trust. (2011). Health Systems Trust Health Statistics. from http://www.hst.org.za/healthstats/ index.php

8. International Telecommunications Union. (2011). Key Global Telecom Indicators for the World Telecommunication Service Sector. from http://www.itu. int/ITU-D/ict/statistics/at_glance/KeyTelecom.html

9. Jithoo, R., Govender, P. V., Corr, P., \& Nathoo, N. (2003). Telemedicine and neurosurgery: experience of a regional unit based in South Africa. J.Telemed.Telecare, 9(2), 6366.

10. Kekana, M., Noe, P., \& Mkhize, B. (2010). The practice of telemedicine and challenges to the regulatory authorities. South African Journal of Bioethics and the Law, 3(1), 3337.

11. Mars, M. (2007). Telemedicine in KwaZulu-Natal, South Africa: from failure to cautious optimism. Journal of Telemedicine and Telecare, 13(Suppl 3), 57-59.

12. Mars, M. (2009). Tele-education in an African country Global telemedicine and ehealth updates (Vol. 2, pp. 332335). Luxembourg: LuxExpo.

13. Mars, M., \& Jack, C. (2010). Why is telemedicine a challenge to the regulators? South African Journal of Bioethics and the Law, 3(2), 55-58.

14. Mars, M., \& Scott, R. E. (2010). Global e-health policy: a work in progress. Health Affairs, 29(2), 1-8.

15. Minwatts Marketing Group. (2011). Internet usage statistics for Africa. from http://www.internetworldstats. $\mathrm{com} / \mathrm{stats} 1 . \mathrm{htm}$

16. Molefi, M. (2006). A Primary Health Care Telemedicine Workstation. Retrieved from http://www.medetel.lu/ download/2006/parallel_sessions/presentation/0407/ Molefi.pdf

17. Nepad. (2009). The new partnership for Africa's development (NEPAD) health strategy. Initial programme of action. from http://www.chr.up.ac.za/undp/regional/ docs/nepad5.pdf

18. Ontario Hospital Association. (2011). Health systems facts and figures. Retrieved 31 March 2011

19. Simpill. (2011). Simpill. from http://www.simpill.com/ thesimplesolution.html

20. South Africa Info. (2011). Health care in South Africa. from http://www.safrica.info/about/health/health.htm

21. Statistics South Africa. (2010). Mid-year population estimates 2010. Pretoria.

22. United Nations Department of Economic and Social Affairs. (2010). World Urbanization Prospects: the 2009 Revision Population Database. [http://esa.un.org/ wup2009/unup]. 
23. United Nations Department of Economic Social Affairs Population Division. (2009). World population prospects. The 2008 revision highlights. New York: United Nations.

24. United Nations Development Program. (2010). Human Development Report 2010 (Vol. 20). New York: Palgrave Macmillan.

25. World Bank. (2011). World Development Indicators. from http://www.google.com/publicdata?ds=wbwdi\&met=ny_gdp_mktp_cd\&idim=country:ZAF\&dl=en\&h $\mathrm{I}=\mathrm{en} \& \mathrm{q}=\mathrm{gdp}+$ south+africa

26. World Confederation of Physical Therapists. (2011). from http://www.wcpt.org/africa

27. World Federation of Occupational Therapists. (2011). from http://www.wfot.org/

28. World Health Organization. (2006a). Building foundations for eHealth. Report of the WHO Global Observatory for eHealth. Geneva: WHO Press.

29. World Health Organization. (2006b). World health report 2006: working together for health. Geneva: WHO Press.

30. World Health Organization. (2010). World health statistics 2010: WHO Press.

31. World Health Organization African Region. (2011). eHealth solutions in the African region: current context and perspectives. AFR/RC60/R3. Retrieved from http://www. google.co.za/\#hl=en\&safe $=$ off\&biw $=689 \& b i h=539 \& q=e H$ ealth+solutions+in+teh+African+Region\&aq=o\&aqi=\&aql $=\& o q=\& f p=6104$ ee3752daf963 